\title{
Distances between elements of a semigroup and estimates for derivatives
}

\author{
Z. Bendaoud, I. Chalendar $†$ J. Esterle ${ }^{\ddagger}$ and J.R. Partington ${ }^{\S}$
}

January 9, 2009

\begin{abstract}
This paper is concerned first with the behaviour of differences $T(t)-T(s)$ near the origin, where $(T(t))$ is a semigroup of operators on a Banach space, defined either on the positive real line or a sector in the right half-plane (in which case it is assumed analytic). For the non-quasinilpotent case extensions of results in the published literature are provided, with best possible constants; in the case of quasinilpotent semigroups on the half-plane, it is shown that, in general, differences such as $T(t)-T(2 t)$ have norm approaching 2 near the origin. The techniques given enable one to derive estimates of other functions of the generator of the semigroup; in particular, conditions are given on the derivatives near the origin to guarantee that the semigroup generates a unital algebra and has bounded generator.
\end{abstract}

Mathematics Subject Classification (2000): Primary: 47D03, 46J40 Secondary: $46 \mathrm{H} 20$

Abbreviated title: Distances between elements of a semigroup

*Université Amar Telidji, Laboratoire de Sciences Economiques et Sciences de Gestion, BP 37G, Laghouat 03000, Algeria. z.bendaoud@mail.lagh-univ.dz.

${ }^{\dagger}$ I. C. J., UFR de Mathématiques, Université Lyon 1, 43 bld. du 11/11/1918, 69622 Villeurbanne Cedex, France. chalenda@math.univ-lyon1.fr.

${ }^{\ddagger}$ L.A.B.A.G, Université Bordeaux 1, 351 cours de la Libération, 33405 Talence Cedex, France. jean.esterle@math.u-bordeaux1.fr.

${ }^{\S}$ School of Mathematics, University of Leeds, Leeds LS2 9JT, U.K. J.R.Partington@leeds . ac . uk. 


\section{Introduction}

The first subject of this note is the behaviour of expressions such as $T(t)-$ $T(s)$ near the origin, where $(T(t))_{t}$ is a semigroup of operators on a Banach space, defined either on $\mathbb{R}^{+}$, or on a sector

$$
S_{\alpha}=\{z \in \mathbb{C}: \operatorname{Re}(z)>0 \text { and }|\arg (z)|<\alpha\}
$$

in the right-hand complex half-plane $\mathbb{C}^{+}=\{z \in \mathbb{C}: \operatorname{Re}(z)>0\}$; in the case of a sector, we assume further that the semigroup is analytic. The results we shall derive are stronger forms of those of Bendaoud, Esterle and Mokhtari $[1,5]$, in the case of semigroups defined on the positive real line. For example, they show that if

$$
\limsup _{t \rightarrow 0}\|T(t)-T((n+1) t)\|<\frac{n}{(n+1)^{1+1 / n}},
$$

then either the semigroup is trivial, or it generates a unital closed subalgebra $\mathcal{A}$, and indeed $T(t)=\exp (t u)$ for some $u \in \mathcal{A}$. Moreover, this constant is shown to be optimal.

In Section 2, we relax Condition (1) to

$$
\|T(t)-T(t(n+1))\|<\frac{n}{(n+1)^{1+1 / n}},
$$

for all $t$ in an interval $(0, \delta)$. In fact, there are two cases to consider, the easier non-quasinilpotent case, and then the general case. Further, we show that, unlike the results of [5], these results cannot be extended to a result using only $T(t)$ for $t$ in any proper measurable subgroup of $\mathbb{R}$ (for example, $\mathbb{Q})$.

For analytic semigroups defined in a sector $S_{\alpha}$, we shall show in Section 3 that a similar result holds, where the optimal constants are given explicitly in terms of $\alpha$. In fact, these results are put in a much more general framework, 
which enables us to study quantities such as $T^{\prime}(t)=A T(t)$ (the derivative of the semigroup) and indeed linear combinations of elements of the semigroup and its derivatives. In the limiting case of the half-plane, an analogous result is proved by different methods for $T(t)-T((\gamma+1) t)$, although it will be seen that no such result can hold in the more general situation. We conclude by extending and applying results of Hille $[8,9]$ to show that if

$$
\sup _{t \in S_{\alpha}, 0<|t|<\delta}\left\|t^{n} T^{(n)}(t)\right\|<\left(\frac{n}{e \cos (\alpha)}\right)^{n}
$$

for some $\delta>0$, then the closed algebra generated by the semigroup is unital, and the generator of the semigroup is bounded.

\section{Semigroups on $\mathbb{R}^{+}$}

\subsection{The non-quasinilpotent case}

The following result is a strengthening of Theorem 2.3 of [5]:

Theorem 2.1 Let $(T(t))_{t>0}$ be a non-quasinilpotent semigroup in a Banach algebra, let $\mathcal{A}$ be the closed subalgebra generated by $(T(t))_{t>0}$ and let $\gamma>0$ be a real number. If

$$
\rho(T(t)-T(t(\gamma+1)))<\frac{\gamma}{(\gamma+1)^{1+\frac{1}{\gamma}}}
$$

for $0<t \leq t_{0}$, for some $t_{0}>0$, then $\mathcal{A} / \operatorname{Rad}(\mathcal{A})$ is unital, and there exist an idempotent $J$ in $\mathcal{A}$, an element $u$ of $J \mathcal{A}$ and a mapping $r: \mathbb{R}^{+} \rightarrow \operatorname{Rad}(J \mathcal{A})$, with the following properties:

(i) $\phi(J)=1$ for all $\phi \in \widehat{\mathcal{A}}$;

(ii) $r(s+t)=r(s)+r(t)$ for all $s, t \in \mathbb{R}^{+}$;

(iii) $J T(t)=e^{t u+r(t)}$ for $t \in \mathbb{R}^{+}$, where $e^{v}=J+\sum_{k \geq 1} \frac{v^{k}}{k !}$ for $v \in J \mathcal{A}$;

(iv) $(T(t)-J T(t))_{t \in \mathbb{R}^{+}}$is a quasinilpotent semigroup. 
Proof : $\quad$ Let $\phi \in \widehat{\mathcal{A}}$; then, by Lemma 2.1 of [5] there exists $c \in \mathbb{C}$ such that $\phi(T(t))=e^{c t}$ for all $t>0$. We have

$$
\begin{aligned}
\left.|| \phi(T(t))|-| \phi(T(t))\right|^{\gamma+1} \mid & \leq\left|\phi(T(t))-\phi(T(t))^{\gamma+1}\right| \\
& <\frac{\gamma}{(\gamma+1)^{1+\frac{1}{\gamma}}} \text { for } t \in\left(0, t_{0}\right] .
\end{aligned}
$$

Then either $\quad|\phi(T(t))|<\frac{1}{(\gamma+1)^{\frac{1}{\gamma}}}, \quad$ or else $\quad|\phi(T(t))|>\frac{1}{(\gamma+1)^{\frac{1}{\gamma}}}$,

and the set $(|\phi(T(t))|)_{0<t \leq t_{0}}$ is an interval $I$ which does not contain $\frac{1}{(\gamma+1)^{\frac{1}{\gamma}}}$. Since $\lim _{t \rightarrow 0^{+}} \phi(T(t))=1$, we have

$$
I \subset\left(\frac{1}{(\gamma+1)^{\frac{1}{\gamma}}},+\infty\right),
$$

and $\mid \phi\left(T\left(t_{0}\right) \mid>\frac{1}{(\gamma+1)^{\frac{1}{\gamma}}}\right.$ for all $\phi \in \widehat{\mathcal{A}}$.

Thus $\widehat{\mathcal{A}}$ is compact. By Theorems 3.6.3 and 3.6.6 of [11], the quotient algebra $\mathcal{A} / \operatorname{Rad}(\mathcal{A})$ is unital, there exists an idempotent $J$ in $\mathcal{A}$ such that $\phi(J)=1$ for all $\phi \in \widehat{\mathcal{A}}$, and $(T(t)-J T(t))_{t \in \mathbb{R}^{+}}$is a quasinilpotent semigroup.

Consider the algebra $\mathcal{B}=J \mathcal{A}$ with unit $J$, and define $S(t)=J T(t)$ for $t \in \mathbb{R}^{+}$. Let $\psi \in \widehat{\mathcal{B}}$; the mapping $\phi: x \rightarrow \psi(J x)$ is a character of $\mathcal{A}$. We have

$$
\begin{aligned}
\frac{1}{(\gamma+1)^{\frac{1}{\gamma}}}|1-\psi(S(t \gamma))| & \leq|\phi(T(t))||1-\psi(S(t \gamma))| \\
& =|\phi(T(t))-\phi(T(t(\gamma+1)))| \leq \frac{\gamma}{(\gamma+1)^{1+\frac{1}{\gamma}}}
\end{aligned}
$$

for $t \in\left(0, t_{0}\right]$. Thus

$$
|1-\psi(S(t \gamma))| \leq \frac{\gamma}{(\gamma+1)}<1
$$


and $\rho(J-S(t))<1$ for $0<t \leq t_{0}$. We write

$$
u_{t}=\sum_{k \geqslant 1} \frac{(-1)^{k+1}(S(t)-J)^{k}}{k} \text { for } t \in\left(0, t_{0}\right] .
$$

We have $S(t)=e^{u_{t}}$. We now extend this expression to $\mathbb{R}^{+}$.

Let $t \in \mathbb{R}^{+}$, and let $n$ be the least positive integer such that $t \leq n t_{0}$. We define $u_{t}=n u_{\frac{t}{n}}$, and then $S(t)=e^{u_{t}}$ for $t \in \mathbb{R}^{+}$.

Now consider once more $\psi \in \widehat{\mathcal{B}}$ and let

$$
\phi: x \rightarrow \psi(J x)
$$

be the character of $\mathcal{A}$ associated with $\psi$. There exists $c \in \mathbb{C}$ such that

$$
\psi(S(t))=\phi(T(t))=e^{c t}
$$

for $t>0$. We have

$$
\psi\left(u_{t}\right)=\sum_{k \geqslant 1} \frac{(-1)^{k+1} \psi(S(t)-J)^{k}}{k},
$$

thus $\psi\left(u_{t}\right)$ coincides with the principal value of the logarithm of

$$
1+\psi(S(t)-J)=\psi(S(t))=e^{c t}
$$

for $0<t \leq t_{0}$. Thus

$$
\psi\left(u_{t}\right)=c t \text { for } 0<t \leq t_{0}
$$

We obtain $\psi\left(u_{t}\right)=n \psi\left(u_{\frac{t}{n}}\right)=c t$ for $t>0$. Let $u=t_{0}^{-1} u_{t_{0}}$ and $r(t)=u_{t}-t u$.

We have

$$
\psi(r(t))=c t-c t=0,
$$

thus $r(t) \in \operatorname{Rad}(\mathcal{A})$ for $t \in \mathbb{R}^{+}$; also

$$
e^{r(s)+r(t)}=e^{-(s+t) u} S(s+t)=e^{r(s+t)} .
$$


Since the mapping $x \rightarrow e^{x}$ is injective on $\operatorname{Rad}(\mathcal{A})$, it follows from Lemma 2.2 of [5] that we have

$$
r(s+t)=r(s)+r(t)
$$

for $s, t \in \mathbb{R}^{+}$.

We deduce the following corollary:

Corollary 2.2 Let $(T(t))_{t \in \mathbb{R}^{+}}$be a non-trivial semigroup in a commutative semi-simple Banach algebra, let $\mathcal{A}$ be the closed subalgebra generated by $(T(t))_{t \in \mathbb{R}^{+}}$and let $\gamma>0$. If

$$
\rho(T(t)-T((\gamma+1) t))<\frac{\gamma}{(\gamma+1)^{1+\frac{1}{\gamma}}},
$$

then $\mathcal{A}$ is unital and there exists an element $u \in \mathcal{A}$ such that $T(t)=e^{t u}$ for $t \in \mathbb{R}^{+}$.

\subsection{The general case}

We begin with a preliminary result about quasinilpotent semigroups. Note that the lemma remains true if we replace $\mathbb{R}^{+}$by $K^{+}:=K \cap \mathbb{R}^{+}$, where $K$ is a subfield of $\mathbb{R}$.

Lemma 2.3 Let $(T(t))_{t \in \mathbb{R}^{+}}$be a quasinilpotent semigroup, and let $n \geq 1$ be an integer. If

$$
\|T(t)-T(t(n+1))\|<\frac{n}{(n+1)^{1+\frac{1}{n}}}
$$

for $t \in\left(0, t_{0}\right]$ for some $t_{0}>0$, then $T(t)=0$ for $t \in \mathbb{R}^{+}$.

Proof : Let $t_{0}>0$. If $(T(t))_{t>0}$ is non-zero, then there exists $t_{1} \in\left(0, t_{0}\right]$ such that $T\left(t_{1}\right) \neq 0$. For $p \geq 1$ we have

$$
\left\|T\left(t_{1} / p\right)\right\| \geq\left\|T\left(t_{1}\right)\right\|^{\frac{1}{p}}, \quad \text { and } \quad\left\|T\left(t_{1} / p\right)\right\| \geq \frac{1}{(n+1)^{\frac{1}{n}}}
$$


for $p$ sufficiently large. It follows therefore from Theorem 4.4 of [5] that

$$
\left\|T\left(\frac{t_{1}}{p}\right)-T\left(\frac{t_{1}(n+1)}{p}\right)\right\|>\frac{n}{(n+1)^{1+\frac{1}{n}}},
$$

which is a contradiction. Hence $T(t)=0$ for $t \in \mathbb{R}^{+}$.

The following result appears implicitly in the proof of Theorem 3.2 of [5].

Lemma 2.4 Let $\mathcal{A}$ be a Banach algebra and let $\theta:(0,+\infty) \rightarrow \mathcal{A}$ satisfy

$$
\theta(s+t)=\theta(s)+\theta(t) \text { for } s>0, t>0 .
$$

If $\limsup _{t \rightarrow 0^{+}}\|\theta(t)\|<+\infty$ then $\theta(t)=t \theta(1)$ for $t>0$.

We employ Lemma 2.4 in the following result.

Lemma 2.5 Let $(T(t))_{t>0}$ be a non-quasinilpotent semigroup in a Banach algebra. Suppose that there exist $\gamma>0$ and $t_{0}>0$ such that

$$
\|T(t)-T(t(\gamma+1))\|<\frac{\gamma}{(\gamma+1)^{1+\frac{1}{\gamma}}}
$$

for $0<t<t_{0}$. Let $J$ be the idempotent in the closed subalgebra $\mathcal{A}$ generated by the semigroup satisfying Properties (i)-(iv) of Theorem 2.1; then there exists $v \in J \mathcal{A}$ such that $J T(t)=e^{t v}$ for $t>0$.

Proof : $\quad$ Let $U$ be the open disc centred at 0 and with radius $\frac{\gamma}{(\gamma+1)^{1+\frac{1}{\gamma}}}$, and let $g: U \rightarrow \mathbb{C}$ be the holomorphic function on $U$ satisfying $g(0)=0$ and

$$
e^{g(z)}-e^{(\gamma+1) g(z)}=z
$$

for $z \in U$. Let

$$
b_{t}=J T(t)-J T(t(\gamma+1)) \text { for } t>0 .
$$

Since

$$
\left\|b_{t}^{k}\right\| \leq\|J\|\left(\frac{\gamma}{(\gamma+1)^{1+\frac{1}{\gamma}}}\right)^{k} \text { for } k>1
$$


the series

$$
\sum_{k \geq 1} \frac{g^{(k)}(0)}{k !}\left\|b_{t}^{k}\right\|
$$

converges. Write

$$
g\left(b_{t}\right):=\sum_{k \geq 1} \frac{g^{(k)}(0)}{k !} b_{t}^{k} .
$$

It follows from standard properties of the holomorphic functional calculus that

$$
e^{g\left(\lambda b_{t}\right)}-e^{(\gamma+1) g\left(\lambda b_{t}\right)}=\lambda b_{t} \text { for } \quad \lambda<1 .
$$

By continuity we obtain, with the notation of Theorem 2.1, that

$$
e^{g\left(b_{t}\right)}-e^{(\gamma+1) g\left(b_{t}\right)}=b_{t}=e^{t u+r(t)}-e^{(\gamma+1)(t u+r(t))} .
$$

For $0<t<t_{0}$ we have

$$
\rho(t u+r(t))=t \rho(u)
$$

thus

$$
\lim _{t \rightarrow 0^{+}} \rho(t u+r(t))=0
$$

and so

$$
\rho\left(b_{t}\right)=\rho\left(e^{t u+r(t)}-e^{(\gamma+1)(t u+r(t))}\right)=\rho\left(e^{t u}-e^{t(\gamma+1) u}\right) \leq e^{t \rho(u)} \rho\left(J-e^{t \gamma u}\right),
$$

from which we conclude that $\lim _{t \rightarrow 0^{+}} \rho\left(b_{t}\right)=0$. Since $g(0)=0$ we have $\lim _{t \rightarrow 0^{+}} \rho\left(g\left(b_{t}\right)\right)=0$.

Let $f: z \mapsto e^{z}-e^{(\gamma+1) z}$, and define

$$
F\left(z_{1}, z_{2}\right)= \begin{cases}\frac{f\left(z_{1}\right)-f\left(z_{2}\right)}{z_{1}-z_{2}} & \text { if } z_{1} \neq z_{2}, \\ f^{\prime}\left(z_{1}\right) & \text { if } z_{1}=z_{2} .\end{cases}
$$

Then $f$ and $F$ are entire functions. Since $f^{\prime}(0) \neq 0$ there is an open disc $D(0, \alpha)$ such that $f$ is injective and $F\left(z_{1}, z_{2}\right) \neq 0$ for $z_{1}, z_{2}$ in $D(0, \alpha)$. Let $t_{1}$ be such that $\rho\left(g\left(b_{t}\right)\right)<\alpha$ for $0<t<t_{1}$. For $t \in\left(0, t_{1}\right]$ we have

$$
0=f\left(g\left(b_{t}\right)\right)-f(t u+r(t))=\left(g\left(b_{t}\right)-(t u+r(t))\right)\left(F\left(g\left(b_{t}, t u+r(t)\right)\right)\right) .
$$


For $\phi \in \widehat{J \mathcal{A}}$ we also have

$$
\phi\left(F\left(g\left(b_{t}\right),(t u+r(t))\right)=F\left(\phi\left(g\left(b_{t}\right), \phi(t u+r(t))\right) \neq 0\right.\right.
$$

and thus $F\left(g\left(b_{t}\right),(t u+r(t))\right)$ is invertible in $J \mathcal{A}$ and

$$
g\left(b_{t}\right)=t u+r(t), \text { for } t \in\left(0, t_{1}\right]
$$

On the other hand, by Lemma 2.1 of [1] we have

$$
\limsup _{t \rightarrow 0^{+}}\left\|g\left(b_{t}\right)\right\| \leq \sum_{k \geq 1} \frac{\left|g^{(k)}(0)\right|}{k !}\left\|b_{t}^{k}\right\|=-\sum_{k \geq 1} \frac{g^{(k)}(0)}{k !}\left[\frac{\gamma}{(\gamma+1)^{1+\frac{1}{\gamma}}}\right]^{\gamma}<+\infty .
$$

Thus

$$
\limsup _{t \rightarrow 0^{+}}\|r(t)\|<+\infty
$$

and it follows from Lemma 2.4 that $r(t)=\operatorname{tr}(1)$. We therefore have $J T(t)=$ $e^{t v}$ for $t>0$ with $v=u+r(1)$.

We now come to the main theorem of this section, which combines the results in Lemmas 2.3 and 2.5.

Theorem 2.6 Let $(T(t))_{t>0}$ be a non-trivial semigroup in a Banach algebra, let $\mathcal{A}$ be the closed subalgebra generated by $(T(t))_{t>0}$ and let $n \geq 1$ be an integer. If there exists $t_{0}>0$ such that

$$
\|(T(t)-T(t(n+1)))\|<\frac{n}{(n+1)^{1+\frac{1}{n}}}
$$

for $0<t \leq t_{0}$, then $\mathcal{A}$ possesses a unit $J, \lim _{t \rightarrow 0^{+}} T(t)=J$ and there exists $u \in \mathcal{A}$ such that $T(t)=e^{t u}$ for all $t>0$.

Proof : Since

$$
\rho(T(t)-T(t(n+1))) \leq\|T(t)-T(t(n+1))\|
$$


there exists an idempotent $J$ in $\mathcal{A}$ satisfying the conditions of Theorem 2.1. In particular, $S(t):=T(t)-J T(t)$ is quasinilpotent for $t>0$. Let $\pi$ : $\mathcal{A} \rightarrow \mathcal{A} / J \mathcal{A}$ be the canonical surjection. Since $\pi(S(t))_{t>0}$ is a quasinilpotent semigroup in $\mathcal{A} / J \mathcal{A}$, it follows from Lemma 2.3 that

$$
\pi(T(t))=\pi(S(t))=0,
$$

and $T(t) \in J \mathcal{A}$, for $t>0$. Thus $T(t)=J T(t)$. By Lemma 2.5 there exists $v \in \mathcal{A}=J \mathcal{A}$ such that $T(t)=J T(t)=e^{t v}$ for $t>0$.

\subsection{Counterexamples for subgroups of $\mathbb{R}$}

We now show that Theorem 2.6 is sharp, in the sense that analogous results do not hold when we consider certain additive subgroups of the real line. Indeed, we give a counter-example applying to any proper measurable subgroup of $\mathbb{R}$.

Proposition 2.7 Let $G$ be an additive subgroup of $\mathbb{R}$ which is measurable and with $G \neq \mathbb{R}$. Then, given a sequence $\left(\gamma_{n}\right)_{n}$ in $\mathbb{R}^{+}$such that $t \gamma_{n} \in G$ for all $t \in G, t>0$, there exists a semigroup $(S(t))_{t \in G, t>0}$ in $c_{0}$ such that

$$
\left\|S(t)-S\left(t\left(\gamma_{n}+1\right)\right)\right\|<\frac{\gamma_{n}}{\left(\gamma_{n}+1\right)^{1+1 / \gamma_{n}}},
$$

for all $t \in G, t>0$.

Proof : Note that the measure of $G$ is zero, as otherwise $G-G$ contains an interval $(-\delta, \delta)$ with $\delta>0$ (cf. [7, Thm. 16.B]), and then $G=\mathbb{R}$. Let $S(t)=\left(a_{k}^{t}\right)_{k \geq 1}$, where $0<a_{k}<1$ for all $k \geq 1$ and $a_{k} \rightarrow 0$ as $k \rightarrow \infty$. Thus $S(t) \in c_{0}$. Further conditions will be imposed on $a_{k}$ later. Since $a_{k} \rightarrow 0$, we have

$$
\left\|S(t)-S\left(t\left(\gamma_{n}+1\right)\right)\right\|=\max _{k}\left|a_{k}^{t}-a_{k}^{t\left(\gamma_{n}+1\right)}\right| .
$$


Observe that

$$
\left|a^{t}-a^{t\left(\gamma_{n}+1\right)}\right| \leq \frac{\gamma}{(\gamma+1)^{1+1 / \gamma}},
$$

for all $a \in(0,1), t>0$ and $\gamma>0$. Indeed, its maximum value over $t$ occurs when $(\ln a) a^{t}=(\gamma+1)(\ln a) a^{t+t \gamma}$, i.e. $a^{t}=\frac{1}{(1+\gamma)^{1 / \gamma}}$. For this value of $t$ Equation (2) is an equality, otherwise it is a strict inequality. Therefore,

$$
\left\|S(t)-S\left(t\left(\gamma_{n}+1\right)\right)\right\| \leq \frac{\gamma_{n}}{\left(\gamma_{n}+1\right)^{1+1 / \gamma_{n}}}
$$

and provided that $a_{k}^{t} \neq \frac{1}{\left(1+\gamma_{n}\right)^{1 / \gamma_{n}}}$ for all $k$, the inequality in (3) is strict. The set

$$
H=\left\{\left(\frac{1}{1+\gamma_{n}}\right)^{1 / g}: g \in G, g>0, n \geq 1\right\}
$$

is a set of measure 0 . So there exists a sequence $\left(a_{k}\right)_{k}$ in $(0,1)$ tending to 0 and such that $a_{k} \notin H$ for all $k$. With this choice we have

$$
\left\|S(t)-S\left(t\left(\gamma_{n}+1\right)\right)\right\|<\frac{\gamma_{n}}{\left(\gamma_{n}+1\right)^{1+1 / \gamma_{n}}},
$$

for all $t>0$ with $t, t \gamma_{n} \in G$.

Remark 2.8 The same conclusion holds if we assume that $G$ is meagre, since $H$ will also be meagre as a countable union of meagre sets

\section{Sectorial semigroups}

Let $T(t)_{t>0}$ be a strongly continuous semigroup of bounded operators on a Banach space $X$. Denote by $\mathcal{D}_{A}$ the set of all $x \in X$ for which $\frac{T(t) x-x}{t}$ has a limit as $t \rightarrow 0^{+}$. Then $\mathcal{D}_{A}$ is a linear subspace of $X$ which is dense in $\cup_{t>0} T(t) X$, since it contains all vectors of the form $\int_{\alpha}^{\beta} T(s) x d x, x \in X, 0<\alpha<\beta$, and the (infinitesimal) generator of the semigroup is the linear operator $A: \mathcal{D}_{A} \rightarrow A$ defined for $x \in \mathcal{D}_{A}$ by the formula 


$$
A x=\lim _{t \rightarrow 0^{+}} \frac{T(t)-x}{t} .
$$

We will say that the generator of the semigroup $T(t)$ is bounded if there exists $K>0$ such that $\|A x\| \leq K\|x\|$ for every $x \in \mathcal{D}_{A}$. This condition is equivalent to the fact that there exists $P \in \mathcal{B}(X)$ such that $\lim _{t \rightarrow 0^{+}} \| T(t)-$ $P \|=0$. In this situation $\mathcal{D}_{A}=\left[\bigcup_{t>0} T(t) X\right]^{-}$, we can consider $A$ as a bounded operator on $X$ by using the formula $A x:=A P x$ for $x \in X$, and we have, for $x \in X, t>0$,

$$
T(t) x=P e^{t A x}
$$

We thus see that for a strongly continuous semigroup $(T(t))_{t>0}$ of bounded operators on a Banach space $X$, the conclusions of Corollary 2.2, Lemma 2.5 and Theorem 2.6 are equivalent to the fact that the generator of the semigroup is bounded.

Now assume that a semigroup $(T(t))_{t>0}$ of bounded operators on a Banach space $X$ is differentiable on $(0,+\infty)$, which means that

$$
T^{\prime}(t):=\lim _{h \rightarrow 0} \frac{T(t+h)-T(t)}{h}
$$

exists in $(\mathcal{B}(H),\|\|$.$) for every t>0$. Then $\bigcup_{t>0} T(t) X \subset \mathcal{D}_{A}$, and we have, for $t>0$, (see for example $[9,10.3 .6]$

$$
T^{\prime}(t)=A T(t)
$$

More generally if $(T(t))_{t>0}$ is $n$-times continuously differentiable on $(0,+\infty)$, then $\bigcup_{t>0} T(t) X \subset \mathcal{D}_{A^{n}}$, and we have, for $t>0$,

$$
T^{(n)}(t)=A^{n} T(t)
$$

We will say that a semigroup $(T(t))_{t}>0$ of bounded operators on a Banach space $X$ is norm continuous if $\lim _{h \rightarrow 0}\|T(t+h)-T(t)\|=0$ for every $t>0$. Notice that if the closed subalgebra $\mathcal{A}$ of $\mathcal{B}(X)$ generated by a norm 
continuous semigroup $(T(t))_{t>0}$ of bounded operators on a Banach space $X$ is unital, then the generator $A$ of the semigroup is bounded. Indeed there exists in this situation a strictly increasing finite sequence $\left(t_{i}\right)_{1 \leq i \leq n}$ of positive real numbers and a family $\left(\lambda_{i}\right)_{1 \leq i \leq n}$ of nonzero complex numbers such that

$$
\left\|P-\sum_{i=1}^{n} \lambda_{i} T\left(t_{i}\right)\right\|<1,
$$

where $P$ denotes the unit element of $\mathcal{A}$. This shows that

$$
T\left(t_{1}\right)\left[P+\sum_{i=2}^{n} \lambda_{i} T\left(t_{i}-t_{1}\right)\right]=\sum_{i=1}^{n} \lambda_{i} T\left(t_{i}\right)
$$

is invertible in $\mathcal{A}$. Hence $T\left(t_{1}\right)$ is invertible in $\mathcal{A}$, and $P=T\left(t_{1}\right)^{-1} T\left(t_{1}\right)=$ $T\left(t_{1}\right)^{-1} \lim _{h \rightarrow 0^{+}} T\left(t_{1}+h\right)=\lim _{h \rightarrow 0^{+}} T(h)$, and the generator of the semigroup is bounded.

It is more convenient to consider more generally norm continuous semigroups $(T(t))_{t>0}$ in Banach algebras. In this case we can set $X=\mathcal{A}$, where $\mathcal{A}$ is the closed subalgebra generated by the semigroup. Then $\bigcup_{t>0} T(t) \mathcal{A}$, which contains $\bigcup_{t>0} T(t)$, is a dense ideal of $\mathcal{A}$. When $\mathcal{A}$ has a unit element $P$, then the dense ideal $\mathcal{D}_{A}$ equals $A, P \in \mathcal{D}_{A}$ and we see again that $A=\lim _{t \rightarrow 0^{+}} \frac{T(t) P-P}{t}$ is bounded.

We now study the behaviour of $\|T(t)-T((\gamma+1) t)\|$ as $t \rightarrow 0$ in $\mathbb{C}^{+}$when $(T(t))_{t \in \mathbb{C}^{+}}$is analytic on the open right-hand half-plane $\mathbb{C}_{+}$. Later we look at other sectors, by a different method.

First, we require an easy technical lemma

Lemma 3.1 Let $S$ be a sector of $\mathbb{C}^{+}$, say

$$
S=S_{\alpha, \beta}:=\left\{z \in \mathbb{C}^{+}:-\alpha<\arg (z)<\beta\right\}
$$

with $\alpha, \beta>0$, and let $\theta: S \rightarrow \mathbb{C}$ be a nonzero analytic function such that $\Theta(s+t)=\Theta(s) \Theta(t)$ for all $s, t \in S$ such that $s+t \in S$. Then there exists $c \in \mathbb{C}$ such that $\Theta(t)=e^{-c t}$ for all $t \in S$. 
Proof : The result is clear for $t \in \mathbb{Q}^{+}$with $c$ such that $\Theta(1)=e^{-c}$. By continuity and analyticity it is also true on $S$.

Theorem 3.2 Let $\left(T(t)_{t \in \mathbb{C}^{+}}\right.$be an analytic non-quasinilpotent semigroup in a Banach algebra. Let $\mathcal{A}$ be the closed subalgebra generated by $(T(t))_{t \in \mathbb{C}^{+}}$and let $\gamma>0$. If there exists $t_{0}>0$ such that

$$
\left.\sup _{t \in \mathbb{C}^{+},|t| \leq t_{0}} \rho(T(t)-T(\gamma+1) t)\right)<2
$$

then $\mathcal{A} / \operatorname{Rad} \mathcal{A}$, is unital, and the generator of $\left(\pi(T(t))_{t>0}\right.$ is bounded, where $\pi: \mathcal{A} \rightarrow \mathcal{A} / \operatorname{Rad} \mathcal{A}$ denotes the canonical surjection.

Proof : $\quad$ Choose $\Phi \in \widehat{\mathcal{A}}$. By Lemma 3.1, there exists $c(\Phi) \in \mathbb{C}$ such that $\Phi(T(t))=e^{-c(\Phi) t}$ for all $t \in \mathbb{C}^{+}$. Denote by $Q$ the set $\left\{t \in \mathbb{C}^{+},|t|<t_{0}\right\}$. By hypothesis, we have:

$$
\sup _{t \in Q}|T(t)-T(t(\gamma+1))|<2
$$

It follows that $c(\Phi) \notin\left\{\frac{i \pi}{\gamma t},-\frac{i \pi}{\gamma t}: t \in \bar{Q}\right\}$, where $\bar{Q}$ denotes the closure of $Q$. Indeed, if $c t=\frac{i \pi}{\gamma}$ or $c t=-\frac{i \pi}{\gamma}$, then $\left|e^{-c t}-e^{-c(\gamma+1) t}\right|=2$. Therefore, there exists $\delta>0$ such that $|c(\Phi)| \leq \delta$ for all $\Phi \in \widehat{\mathcal{A}}$. It follows that $\left|\Phi\left(T\left(t_{0}\right)\right)\right| \geq e^{-\delta t_{0}}$ for all $\Phi \in \widehat{\mathcal{A}}$, and thus $\widehat{\mathcal{A}}$ is compact. Using Theorem 3.6.3 and Theorem 3.6.6 in [11], we conclude that $\mathcal{A} / \operatorname{Rad} \mathcal{A}$ is unital. Since the algebra generated by the norm-continuous semigroup $\left(\pi(T(t))_{t>0}\right.$ is dense in $\mathcal{A} / \operatorname{Rad} \mathcal{A}$, the generator of this semigroup is bounded.

A semigroup $T(t)$ defined over the positive reals or on a sector is said to be exponentially bounded if there exists $c_{1}>0$ and $c_{2} \in \mathbb{R}$ such that $\|T(t)\| \leq$ $c_{1} e^{c_{2}|t|}$ for every $t$. A classical result of Beurling [2] shows that there exists a universal constant $k$ such that every exponentially bounded weakly measurable semigroup $(T(t))_{t>0}$ of bounded operators satisfying $\lim \sup _{t \rightarrow 0^{+}} \| I-$ 
$T(t) \|=\rho<2$ admits an exponentially bounded analytic extension to a sector $S_{\phi, \phi}$, with $\phi \geq k(2-\rho)^{2}$.

It follows immediately from this result that if a semigroup $(T(t))$ is analytic on a sector $S_{\phi, \psi}$, and if this semigroup does not admit any analytic extension to any sector $S_{\phi_{1}, \psi}$ with $\phi_{1}>\phi$, then we have, for $-\psi<\alpha<\phi$,

$$
\limsup _{t \rightarrow 0^{+}}\left\|I-T\left(t e^{i \alpha}\right)\right\| \geq 2-\sqrt{\frac{\phi-\alpha}{k}} .
$$

Similarly, if this semigroup does not admit any analytic extension to any sector $S_{\phi, \psi_{1}}$ with $\psi_{1}>\psi$, then we have, for $-\psi<\alpha<\phi$,

$$
\limsup _{t \rightarrow 0^{+}}\left\|I-T\left(t e^{i \alpha}\right)\right\| \geq 2-\sqrt{\frac{\psi+\alpha}{k}} .
$$

Now if an analytic semigroup $(T(t))_{t>0}$ on the open half plane has an analytic extension to any larger open sector, then $T(t)$ is invertible in the closed subalgebra generated by the semigroup for some $t>0$, and so the generator of the semigroup is bounded. So the following result is an immediate consequence of Beurling's theorem.

Theorem 3.3 Let $(T(t))_{t \in \mathbb{C}^{+}}$be an analytic semigroup of bounded operators on a Banach space $X$. If the generator of the semigroup is unbounded, then we have, for $-\frac{\pi}{2}<\alpha<\frac{\pi}{2}$,

$$
\limsup _{t \rightarrow 0^{+}}\|I-T(t)\| \geq 2-\sqrt{\frac{\frac{\pi}{2}-|\alpha|}{k}},
$$

where $k$ is Beurling's universal constant.

This suggests that $\sup _{\substack{t \in \mathbb{C}^{+} \\|t| \leq \delta}}\|T(t)-T((\gamma+1) t)\| \geq 2$ for every $\delta>0$ if the generator of a semigroup $(T(t)) t \in \mathbb{C}^{+}$is unbounded. We can prove this for a large class of analytic semigroups. 
Theorem 3.4 Let $(T(t))_{t \in \mathbb{C}^{+}}$be an analytic semigroup in a Banach algebra. Assume that there exists $\eta>0$ and $c \in \mathbb{R}$ such that

$$
\sup _{y \in \mathbb{R}} e^{-c \mid y} \mid\|T(\eta+i y)\|<+\infty .
$$

If the generator of the semigroup is unbounded, then we have, for every $\gamma>0$ and every $\delta>0, \sup _{\substack{t \in C^{+} \\|t| \leq \delta}}\|T(t)-T((\gamma+1) t)\| \geq 2$.

Proof : Let $\mathcal{A}$ be the closed subalgebra generated by $(T(t))_{t>0}$. Since the semigroup is exponentially bounded on a vertical line, and since the generator of the semigroup is unbounded, it follows from an elementary construction of [3], based on a method which goes back to [6], that there exists a Banach space $\left(X,\|\cdot\|_{1}\right)$ and a norm-decreasing homomorphism $\theta:(\mathcal{A},\|\cdot\|) \rightarrow$ $\left(\mathcal{B}(X),\|.\|_{1}\right.$ such that if we set $\tilde{T}(t):=\theta(T(t))$ for $t \in \mathbb{C}^{+}$the following conditions are satisfied

1. there exists $\mu \in \mathbb{R}$ such that $\|T(t)\| \leq e^{\mu|t|}$ for $t \in \mathbb{C}^{+}$,

2. $(\tilde{T}(t))_{t \in \mathbb{C}^{+}}$admits a strongly continuous extension to $\overline{\mathbb{C}^{+}}$such that $\tilde{T}(0)=I_{X}$

3. the generator of $(\tilde{T}(t))_{t>0}$ is unbounded.

Notice that the generator of the semigroup $(\tilde{T}(i t))_{t>0}$ is unbounded, for otherwise there would exist $u \in \mathcal{B}(X)$ such that $\tilde{T}(i t)=e^{t u}$ for $t>0$. Hence we would have $\tilde{T}(i t)=e^{t u}$ for $t \in \mathbb{R}$. The analytic semigroup $\left(e^{-i t u} \tilde{T}(t)\right)_{t \in \mathbb{C}^{+}}$ would be constant on vertical lines, hence constant on $\mathbb{C}^{+}$. Since $\lim _{t \rightarrow 0} \tilde{T}(t) x=$ $x$ for every $x \in X$, we would have $\tilde{T}(t)=e^{-i t u}$ for $t \in \overline{\mathbb{C}^{+}}$, which contradicts the fact that the generator of $(\tilde{T}(t))_{t>0}$ is unbounded. This implies in particular that $\lim \sup _{t \rightarrow 0^{+}}\left\|I_{X}-\tilde{T}(i t)\right\|_{1} \geq 2$.

We have, for $x \in X, \delta>0,0<t \leq \delta$,

$$
\begin{aligned}
\left\|\left(I_{X}-\tilde{T}(i \gamma t)\right) x\right\|_{1} & \leq\|\tilde{T}(-i t)\|_{1}\|(\tilde{T}(i t)-\tilde{T}(i(\gamma+1) t)) x\|_{1} \\
& \leq e^{|\mu| \delta}\|(\tilde{T}(i t)-\tilde{T}(i(\gamma+1) t)) x\|_{1} .
\end{aligned}
$$


Hence, for $t \in(0, \delta]$, we have

$$
\left\|I_{X}-\tilde{T}(i \gamma t)\right\|_{1} \leq e^{|\mu| \delta} \liminf _{\alpha \rightarrow \frac{\pi}{2}^{+}}\left\|\tilde{T}\left(t e^{i \alpha}\right)-\tilde{T}\left((\gamma+1) t e^{i \alpha}\right)\right\|_{1} .
$$

Hence for every $\eta \in(0, \delta]$, we have

$$
\begin{gathered}
2 \leq \limsup _{t \rightarrow 0^{+}}\left\|I_{X}-\tilde{T}(i t)\right\|_{1} \leq e^{|\mu| \eta} \sup _{\substack{t \in \mathbb{C}^{+} \\
|t| \leq \eta}}\|\tilde{T}(t)-\tilde{T}((\gamma+1) t)\|_{1} \\
\leq e^{|\mu| \eta} \sup _{\substack{t \in \mathbb{C}^{+} \\
|t| \leq \delta}}\|T(t)-T((\gamma+1) t)\|,
\end{gathered}
$$

and $\sup _{\substack{t \in \mathbb{C}^{+} \\|t| \leq \delta}}\|T(t)-T((\gamma+1) t)\| \geq 2$

Notice that the argument above shows in fact that

$$
\left.\liminf _{\alpha \rightarrow \frac{\pi}{2}-}\left[\sup _{0<t \leq \delta} \| T(t)-T(\gamma+1) t\right) \|\right] \geq 2
$$

and

$$
\left.\liminf _{\alpha \rightarrow-\frac{\pi}{2}}\left[\sup _{0<\leq \delta} \| T(t)-T(\gamma+1) t\right) \|\right] \geq 2
$$

for every analytic semigroup with unbounded generator which admits exponential growth on a vertical line. More precise lower estimates for the distance near the origin of elements of such a semigroup in the quasinilpotent case are given in [3].

Remark 3.5 1. In Theorem 3.2, 2 is the best constant and moreover the limsup cannot be replaced by a pointwise condition, contrary to [1], as shown by the following example:

Let $S(t): x \mapsto x^{t}$ for $x \in[0,1]$ with $t \in \mathbb{C}^{+}$. The function $S(t)$ belongs to $C_{0}([0,1])$, the Banach algebra of continuous functions on $[0,1]$ vanishing at 0 , equipped with the supremum norm. Obviously

$$
\|S(t)-S((\gamma+1) t)\|_{\infty} \leq 2
$$


because $\|S(t)\|_{\infty} \leq 1$ since $\operatorname{Re}(t)>0$. For $t=i \frac{\pi}{\gamma \log x}$, note that $|S(t)(x)-S((\gamma+1) t)(x)|$ is equal to

$$
\left|e^{i \pi / \gamma}-e^{i \pi(\gamma+1) / \gamma}\right|=2 .
$$

In this case we have

$$
\limsup _{t \rightarrow 0, t \in \mathbb{C}^{+}}\|S(t)-S((\gamma+1) t)\|_{\infty}=\rho(S(t)-S((\gamma+1) t))=2,
$$

but the conclusion of Theorem 3.2 is not satisfied, since $C_{0}([0,1])$ is a Banach algebra without any idempotent element.

2. If the semigroup is not holomorphic, Esterle and Mokhtari [5, Ex. 2.5] proved that $1 / 4$ is the best constant (consider $S(x+i y)$ of the form $S(x+i y)=S(x)$ and use their example $S(x)(w)=w^{x}$, providing a semigroup on $\mathbb{R}^{+}$with elements in $\left.C[0,1]\right)$.

We now consider similar results on smaller sectors than the half-plane, and in fact the result we prove will be stated in a far more general context.

Theorem 3.6 Let $0<\alpha<\pi / 2$, and let

$$
S_{\alpha}=\{z \in \mathbb{C}: \operatorname{Re}(z)>0 \text { and }|\arg (z)|<\alpha\} .
$$

Let $f$ be an entire function with $f(0)=0$ and $f(\mathbb{R}) \subseteq \mathbb{R}$, such that

$$
\sup _{\operatorname{Re} z>r}|f(z)| \rightarrow 0 \quad \text { as } \quad r \rightarrow \infty
$$

and $f$ is a linear combination of functions of the form $z^{m} \exp (-z w)$ for $m=0,1,2, \ldots$ and $w \in S_{\alpha}$. Let $(T(t))_{t \in S_{\alpha}}=(\exp (-t A))_{t \in S_{\alpha}}$ be an analytic non-quasinilpotent semigroup in a Banach algebra and let $\mathcal{A}$ be the subalgebra generated by $(T(t))_{t \in \mathbb{C}^{+}}$. If there exists $t_{0}>0$ such that

$$
\sup _{t \in S_{\alpha},|t| \leq t_{0}} \rho(f(t A))<k\left(S_{\alpha}\right),
$$

with $k\left(S_{\alpha}\right)=\sup _{t \in S_{\alpha}}|f(z)|$, then $\mathcal{A} / \operatorname{Rad} \mathcal{A}$ is unital and the generator of $\pi(T(t))_{t \in S_{\alpha}}$ is bounded, where $\pi: \mathcal{A} \rightarrow \mathcal{A} / \operatorname{Rad}(\mathcal{A})$ denotes the canonical surjection. 
Note that $f(t A)$ is well-defined by means of $T(t)$ and its derivatives.

Proof : $\quad$ By the maximum principle, for each $\theta \in(-\pi / 2, \pi / 2), f$ attains its maximum absolute value $M_{\theta}$, say, on the ray $R_{\theta}=\{z: \arg z=\theta\}$ and $M_{\theta}$ is an increasing function of $\theta$ on $[0, \pi / 2)$. So, indeed, $M_{\theta}=k\left(S_{\theta}\right)$ for $\theta \in(0, \pi / 2)$. Moreover, $M_{\theta}=M_{-\theta}$.

Clearly there exists a $d>0$ such that the maximum value of $f$ on each ray $R_{\theta}$ is attained at a point $z$ such that $\operatorname{Re} z \leq d$.

By Lemma 3.1, the hypotheses of the theorem imply that for each $\Phi \in \widehat{\mathcal{A}}$ there exists $c(\Phi) \in \mathbb{C}$ such that $\Phi(T(t))=e^{-c(\Phi) t}$ for all $t \in S_{\alpha}$, and hence $\Phi(f(t A))=f(c(\Phi) t)$. Moreover, we know that

$$
|f(c(\Phi) t)|<k\left(S_{\alpha}\right)
$$

for all $t \in S_{\alpha}$ with $|t| \leq t_{0}$.

If for any point $t$ in the sector $\left\{t \in S_{\alpha}:|t| \leq t_{0}\right\}$ we have $\operatorname{Re} c(\Phi) t>d$, and $|\arg c(\Phi) t| \geq \alpha$, then

$$
|f(\lambda c(\Phi) t)| \geq k\left(S_{\alpha}\right)
$$

for some real $\lambda$ between 0 and 1 , giving a contradiction. Thus if $\left|\arg c(\Phi) t_{0}\right| \geq$ $\alpha$, then $\operatorname{Re} c(\Phi) t_{0} \leq d$.

Now suppose that $0 \leq \beta=\arg c(\Phi) t_{0}<\alpha$ (the other case is similar); then we know that $\operatorname{Re}\left(c(\Phi) t_{0} e^{i(\alpha-\beta)}\right) \leq d$; writing $c(\Phi) t_{0}=r e^{i \beta}$ and $c(\Phi) t_{0} e^{i(\alpha-\beta)}=r e^{i \alpha}$, we deduce that $\operatorname{Re} c(\Phi) t_{0} \leq d \cos \beta / \cos \alpha$. Hence, in all cases, we obtain $\operatorname{Re} c(\Phi) t_{0} \leq d / \cos \alpha$ and

$$
\left|\Phi\left(T\left(t_{0}\right)\right)\right| \geq \exp (-d / \cos \alpha)
$$

and this holds for every $\Phi \in \widehat{\mathcal{A}}$. As in the proof of Theorem 3.2, it follows that $\mathcal{A} / \operatorname{Rad} \mathcal{A}$ is unital and the generator of $\pi(T(t))_{t \in S_{\alpha}}$ is bounded. 
Remark 3.7 Suitable choices of $f(z)$ are linear combinations of $z^{m} \exp (-z)$, $m=1,2,3, \ldots$, and $\exp (-z)-\exp (-(\gamma+1) z)$; also indeed real linear combinations of the form $\sum_{k=1}^{n} a_{k} \exp \left(-b_{k} z\right)$ with $b_{k}>0$ and $\sum_{k=1}^{n} a_{k}=0$. This provides results analogous to those of [10, Thm. 4.12], where the behaviour of expressions such as $\|t A \exp (-t A)\|$ and $\|\exp (-t A)-\exp (-s t A)\|$ was considered for all $t>0$.

Remark 3.8 Another function considered in [10] is $f(z)=e^{-s z} \sin z$, where we now require $s>\tan \alpha$ for $f(t A)$ to be well-defined for $t \in S_{\alpha}$. This does not satisfy the condition (4), but we note that it holds for $z \in S_{\alpha}$, while for $z \notin S_{\alpha}$ there exists a constant $C>0$ such that for each $z$ with $\operatorname{Re} z>C$ there exists $\lambda \in(0,1)$ such that $|f(\lambda z)| \geq \sup _{z \in S_{\alpha}}|f(z)|$. Using this observation, it is not difficult to adapt the proof of Theorem 3.6 to this case.

Remark 3.9 As before, the sharpness of the constants can be shown by considering examples in $C_{0}([0,1])$.

One particular case of the above is used in the estimates considered by Bendaoud, Esterle and Mokhtari [1, 5].

Corollary 3.10 Let $\gamma>0,0<\alpha<\pi / 2$, and

$$
S_{\alpha}=\{z \in \mathbb{C}: \operatorname{Re}(z)>0 \text { and }|\arg (z)|<\alpha\}
$$

Let $(T(t))_{t \in S_{\alpha}}$ be an analytic non-quasinilpotent semigroup in a Banach algebra and let $\mathcal{A}$ be the subalgebra generated by $(T(t))_{t \in \mathbb{C}^{+}}$. If there exists $t_{0}>0$ such that

$$
\sup _{t \in S_{\alpha},|t| \leq t_{0}} \rho(T(t)-T(t(\gamma+1)))<k\left(S_{\alpha}\right),
$$

with $k\left(S_{\alpha}\right)=\sup _{t \in S_{\alpha}}|\exp (-t)-\exp (-(\gamma+1) t)|$, then $\mathcal{A} / \operatorname{Rad} \mathcal{A}$ is unital and the generator of $\pi(T(t))_{t \in S_{\alpha}}$ is bounded, where $\pi: \mathcal{A} \rightarrow \mathcal{A} / \operatorname{Rad}(\mathcal{A})$ denotes the canonical surjection. 
Proof : This follows immediately from Theorem 3.6 on taking $f(z)=$ $\exp (-z)-\exp (-z(\gamma+1))$.

Remark 3.11 1. As in Remark 3.5, it is easy to write down examples in $C_{0}([0,1])$ which show that the constant $M_{\alpha}=k\left(S_{\alpha}\right)$ is sharp.

2. The constant $M_{\alpha}=k\left(S_{\alpha}\right)$ initially grows rather slowly with $\alpha$ : numerical calculations with $\gamma=1$ give $M_{0}=0.25, M_{\pi / 12}=0.26, M_{\pi / 6}=$ $0.29, M_{\pi / 4}=0.35, M_{\pi / 3}=0.47, M_{5 \pi / 12}=0.80$ and $M_{11 \pi / 24}=1.18$. Nonetheless, $M_{\alpha} \rightarrow 2$ as $\alpha \rightarrow \pi / 2$.

3. For sectors $S_{\alpha, \beta}$ that are not symmetric it is clear that a similar result holds with the constant $k\left(S_{\alpha, \beta}\right)=\max \left(k\left(S_{\alpha}\right), k\left(S_{\beta}\right)\right)$.

The same results hold for norm-continuous semigroups $(T(t))_{t>0}$ satisfying $\lim \sup _{t \rightarrow 0^{+}}\|f(t A)\|<k_{0}:=\max _{t>0}|f(t)|$.

These results suggest that if an analytic semigroup $(T(t))_{t \in S_{\alpha}}$ satisfying $\sup _{t \in S_{\alpha}, 0 \leq t \leq \delta}\|f(A)\|<k_{0}$ for some $\delta>0$, then the generator of the semigroup is bounded. We do not know whether this property holds in general, but we will prove it in the special case of functions of the form $f(z)=z^{n} e^{-z}$, where $n \geq 1$ is an integer. We will use a well-known result proved in 1950 by Hille [8] (see also [9, Thm. 10.3.6]). This result is usually stated for $n=1$, but Hille's argument works for any positive integer.

Theorem 3.12 Let $(T(t))_{t>0}$ be a n-times continuously differentiable semigroup over the positive reals. If $\lim \sup _{t \rightarrow 0^{+}}\left\|t^{n} T^{(n)}(t)\right\|<\left(\frac{n}{e}\right)^{n}$, then the generator of the semigroup is bounded.

Proof : Fix $a>0$. We have, for $p \geq 1$, by Taylor's formula

$$
T(t)=\sum_{k=0}^{p-1} \frac{(t-a)^{k}}{k !} T^{(k)}(a)+\frac{1}{(p-1) !} \int_{a}^{t}(t-s)^{p-1} T^{(p)}(s) d s .
$$


Set $\beta=\frac{1}{2}\left(\left[\lim \sup _{t \rightarrow 0^{+}}\left\|t^{n} T^{(n)}(t)\right\|\right]^{1 / n}+\frac{n}{e}\right)$, set $\lambda=\frac{e \beta}{n} \in(0,1)$, and let $\delta>0$ such that $t\left\|T^{(n)}\right\|^{1 / n} \leq \beta$ for $t \in(0, \delta]$. We have, for $q \geq 1, s \in(0, \delta]$,

$$
T^{(n q)}(s)=A^{n q} T(s)=\left[A^{n} T(s / q)\right]^{q}=\left[T^{(n)}(s / q)\right]^{q},\left\|T^{(n q)}(s)\right\| \leq \lambda^{n q} \frac{(n q)^{n q}}{(e s)^{n q}} .
$$

We obtain, for $a \in(0, \delta], t \in(0, \delta]$,

$$
\begin{gathered}
\left\|\frac{1}{(n q-1) !} \int_{a}^{t}(t-s)^{n q-1} T^{(n q)}(s) d s\right\| \leq \frac{\lambda^{n q}(n q)^{n q}}{e^{n q}(n q-1) !} \frac{1}{\min (a, t)^{n q}}\left|\int_{a}^{t}\right| t-\left.s\right|^{n q-1} d s \mid \\
=\left[\frac{\lambda|t-a|}{\min (a, t)}\right]^{n q} \frac{(n q)^{n q}}{e^{n q}(n q) !} .
\end{gathered}
$$

It follows then from Stirling's formula that

$$
\lim _{q \rightarrow+\infty}\left\|T(t)-\sum_{k=0}^{n q-1} \frac{(t-a)^{k}}{k !} T^{(k)}(a)\right\|=0
$$

if $|t-a|<\min \left(\delta-a, \frac{\lambda+1}{a}\right)$.

Now consider again $a \in(0, \delta)$, let $\mu \in(0,1)$, and let $k \geq n$ be an integer. We have $k=n q+r$, with $q \geq 1,0 \leq r \leq n-1$, and we obtain

$T^{(k)}(a)=A^{n q+r} T(a)=\left[A^{n} T\left(\frac{\mu a}{q}\right)\right]^{q} \cdot A^{r} T((1-\mu) a)=\left[T^{(n)}\left(\frac{\mu a}{q}\right)\right]^{q} T^{(r)}((1-\mu) a)$.

Set $m=\max _{0 \leq r \leq n-1} \| T^{(r)}((1-\mu) a)$. We obtain, for $k \geq n$,

$$
\begin{gathered}
\left\|\frac{T^{(k)}(a)}{k !}\right\| \leq \frac{m}{(n q+1) \ldots(n q+r)} \frac{1}{(n q) !}\left\|T^{(n)}\left(\frac{\mu a}{q}\right)\right\|^{q} \\
\leq \frac{m}{(k-n+1)^{n-1}}\left(\frac{\lambda}{\mu a}\right)^{n q} \cdot\left(\frac{n q}{e}\right)^{n q} \cdot \frac{1}{(n q) !}=\frac{m\left(1+\epsilon_{k}\right)}{\sqrt{2 \pi n q}(k-n+1)^{n-1}}\left(\frac{\lambda}{\mu a}\right)^{n q},
\end{gathered}
$$

with $\lim _{k \rightarrow \infty} \epsilon_{k}=0$.

Hence $\lim \sup _{k \rightarrow+\infty}\left\|\frac{T^{(k)}(a)}{k !}\right\|^{\frac{1}{k}} \leq \frac{\lambda}{\mu a}$ for every $\mu \in(0,1)$, which gives 


$$
\limsup _{k \rightarrow+\infty}\left\|\frac{T^{(k)}(a)}{k !}\right\|^{\frac{1}{k}} \leq \frac{\lambda}{a}<\frac{1}{a} .
$$

So the radius of convergence $R$ of the series $\sum_{k=0}^{+\infty} z^{k} \frac{T^{k}(a)}{k !}$ satisfies $R \geq \frac{a}{\lambda}$, and the series converges for $t \in\left(a-\frac{a}{\lambda}, a+\frac{a}{\lambda}\right)$. But it follows from the discussion above that the map $t \rightarrow T(t)$ is analytic on $(0, \delta)$, and so we have, for $t \in(0, \delta)$,

$$
T(t)=\sum_{k=0}^{+\infty} \frac{(t-a)^{k}}{k !} T^{(k)}(a) .
$$

Hence we have

$$
\lim _{t \rightarrow 0^{+}}\left\|T(t)-\sum_{k=0}^{+\infty} \frac{(-a)^{k}}{k !} T^{(k)}(a)\right\|=0,
$$

which shows that the generator of the semigroup is bounded.

Now set $f_{n}(z)=z^{n} e^{-z}$, and set $k_{n}(\alpha)=\max _{z \in S_{\alpha}} f_{n}(z)$. An immediate computation shows that $k_{n}(\alpha)=\left(\frac{n}{e \cos (\alpha)}\right)^{n}$.

If $A$ is the generator of a semigroup $(T(t))_{t \in S_{\alpha}}$ analytic on $S_{\alpha}$, we have $f_{n}(t A)=t^{n} T^{(n)}(t)$. So the following result means that if $\sup _{t \in S_{\alpha}, 0<|t|<\delta}\left\|f_{n}(t A)\right\|<$ $k_{n}(\alpha)$ for some $\delta>0$, then the generator of the semigroup is bounded.

Theorem 3.13 Let $n \geq 1$ be an integer, let $\alpha \in(0, \pi / 2)$ and let $(T(t))_{t \in S_{\alpha}}$ be a semigroup analytic on $S_{\alpha}$. If $\sup _{t \in S_{\alpha}, 0<|t|<\delta}\left\|t^{n} T^{(n)}(t)\right\|<\left(\frac{n}{e \cos (\alpha)}\right)^{n}$ for some $\delta>0$, then the closed algebra generated by the semigroup is unital, and the generator of the semigroup is bounded.

Proof : $\quad$ Let $\beta \in(0, \alpha)$ such that $\left(\frac{n}{e \cos (\beta)}\right)^{n}>\sup _{t \in S_{\alpha}, 0<|t|<\delta}\left\|t^{n} T^{(n)}(t)\right\|$. Set

$$
\begin{aligned}
R(t) & =\left([ t e ^ { i \beta } ] ^ { n } T ^ { ( n ) } ( t e ^ { i \beta } ) \left(\left[t e^{-i \beta}\right]^{n} T^{(n)}\left(t e^{-i \beta}\right)\right.\right. \\
& =t^{2 n} A^{2 n} T\left(t e^{i \beta}+t e^{-i \beta}\right)=t^{2 n} T^{(2 n)}(2 t \cos (\beta)) .
\end{aligned}
$$


We have, for $t \in(0, \beta]$,

$$
\begin{aligned}
\left\|t^{2 n} T^{(2 n)}(2 t \cos (\beta))\right\| & \left.\left.\leq \|\left[t e^{i \beta}\right]^{n} T^{(n)}\left(t e^{i \beta}\right)\right] \|\left[t e^{-i \beta}\right]^{n} T^{(n)}\left(t e^{-i \beta}\right)\right] \\
& <\left(\frac{n}{e \cos (\beta)}\right)^{2 n}=\left(\frac{2 n}{2 e \cos (\beta)}\right)^{2 n} .
\end{aligned}
$$

Hence

$$
\|\left(2 t \cos (\beta)^{2 n} T^{(2 n)}(2 t \cos (\beta)) \|<\left[\frac{2 n}{e}\right]^{2 n}\right.
$$

for every $t \in(0, \delta]$, limsup $\sup _{t \rightarrow 0^{+}}\left\|t^{2 n} T^{(2 n)}(t)\right\|<\left[\frac{2 n}{e}\right]^{2 n}$, and the result follows from Hille's theorem applied to the derivative of order $2 n$ of the given semigroup.

The third author showed in [4] that if $(T(t))_{t>0}$ is a nontrivial strongly continuous quasinilpotent semigroup, then there exists $\delta>0$ such that we have, for $0<t<s \leq \delta$,

$$
\|T(t)-T(s)\|>(s-t) \frac{s^{\frac{s}{s-t}}}{t^{\frac{t}{s-t}}} .
$$

It is easy to deduce from this result that $\left\|t T^{\prime}(t)\right\| \geq \frac{1}{e}$ for $t \in(0, \delta]$ if $(T(t))_{t>0}$ is a quasinilpotent differentiable semigroup. In the other direction it does not seem that the inequality from [4] can be deduced from Hille's theorem, which implies a weaker inequality.

\section{Acknowledgement}

Two of the authors (IC and JRP) are grateful to the EPSRC for financial support.

\section{References}

[1] Z. Bendaoud, J. Esterle, and A. Mokhtari. Distances entre exponentielles et puissances d'éléments de certaines algèbres de Banach. Arch. Math. (Basel), 89(3):243-253, 2007. 
[2] A. Beurling. On analytic extension of semigroups of operators. J. Functional Analysis, 6:387-400, 1970.

[3] I. Chalendar, J. Esterle, and J.R. Partington. Boundary values of sectorial semigroups. Preprint, 2008.

[4] J. Esterle. Distance near the origin between elements of a strongly continuous semigroup. Ark. Mat., 43(2):365-382, 2005.

[5] J. Esterle and A. Mokhtari. Distance entre éléments d'un semi-groupe dans une algèbre de Banach. J. Funct. Anal., 195(1):167-189, 2002.

[6] W. Feller. On the generation of unbounded semi-groups of bounded linear operators. Ann. of Math. (2), 58:166-174, 1953.

[7] P. R. Halmos. Measure Theory. D. Van Nostrand Company, Inc., New York, N. Y., 1950.

[8] E. Hille. On the differentiability of semi-group operators. Acta Sci. Math. Szeged, 12:19-24, 1950.

[9] E. Hille and R. S. Phillips. Functional analysis and semi-groups. American Mathematical Society, Providence, R. I., 1974. Third printing of the revised edition of 1957, American Mathematical Society Colloquium Publications, Vol. XXXI.

[10] N. Kalton, S. Montgomery-Smith, K. Oleszkiewicz, and Y. Tomilov. Power-bounded operators and related norm estimates. J. London Math. Soc. (2), 70(2):463-478, 2004.

[11] C. E. Rickart. General theory of Banach algebras. The University Series in Higher Mathematics. D. van Nostrand Co., Inc., Princeton, N.J.-Toronto-London-New York, 1960. 